\title{
Assessing medical residents' usage and perceived needs for personal digital assistants
}

\author{
James R. Barrett ${ }^{\mathrm{a}, *}$, Scott M. Strayer ${ }^{\mathrm{a}, \mathrm{b}}$, Jane R. Schubart ${ }^{\mathrm{a}}$ \\ a Department of Health Evaluation Sciences, University of Virginia, P.O. Box 800717, \\ Charlottesville, VA 22908, USA \\ ${ }^{\mathrm{b}}$ Department of Family Medicine, University of Virginia, Charlottesville, VA 22908, USA
}

Received 21 August 2003; received in revised form 1 December 2003; accepted 2 December 2003

\section{KEYWORDS}

Hand-held device;

Personal digital

assistant;

Medical resident

training
Summary Health care professionals need information delivery tools for accessing information at the point of patient care. Personal digital assistants (PDAs), or hand-held devices demonstrate great promise as point of care information devices. An earlier study [The Constellation Project: experience and evaluation of personal digital assistants in the clinical environment, in: Proceedings of the 19th Annual Symposium on Computer Applications in Medical Care, 1995, 678] on the use of PDAs at the point of care found that hardware constraints, such as memory capability limited their usefulness, however, they were used frequently for accessing medical references and drug information [The Constellation Project: experience and evaluation of personal digital assistants in the clinical environment, in: Proceedings of the 19th Annual Symposium on Computer Applications in Medical Care, 1995, 678]. Since this study was completed in 1995, hand-held computer technology has advanced rapidly, and between 26 and $50 \%$ of physicians currently use PDAs [Physician's use of hand-helds increases from 15\% in 1999 to 26\% in 2001: Harris interactive poll results, Harris Poll. 8-24-2002 (electronic citation); ACP-ASIM survey finds nearly half of U.S. members use hand-held computers: ACP-ASIM press release, American College of Physicians, 9-3-2002 (electronic citation)]. This use appears higher among residents, with one recent study finding that over two-thirds of family practice residencies use hand-held computers in their training programs [J. Am. Med. Inform. Assoc. 9 (1) (2002) 80].

In this study, we systematically evaluate PDA usage by residents in our institution using quantitative and qualitative methods. Our evaluation included a brief on-line survey of 88 residents in seven residency programs including primary care and specialty practices. The surveys were completed between 26 October 2001 and 30 April 2002. Follow-up interviews with 15 of the surveyed residents were then conducted between 24 April 2002 and 13 May 2002. The original contributions of this study are the evaluation of residents in primary and specialty programs and evaluation of both medical application software and the conventional personal organizational software (such as calendars and to-do lists). This evaluation was also conducted using significantly advanced hardware and software compared with previous studies [The Constellation Project: experience and evaluation of personal digital assistants in the clinical environment, in: Proceedings of the 19th Annual Symposium on Computer Applications in Medical Care, 1995, 678]. 
Results of our survey and follow-up interviews of residents showed most residents use PDAs daily, regardless of practice or whether their program encourages PDAs. Uses include commercial medical references and personal organization software, such as calendars and address books. Concerns and drawbacks mentioned by these residents included physical size of the PDA and the potential for catastrophic data loss. Another issue raised by our results suggests that security and Health Information Portability and Accountability Act (HIPAA) compliance need to be addressed, in part by resident education about securing patient data on PDAs. Overall, PDAs may become even more widely used if two issues can be addressed: (a) providing secure clinical data for the current patients of a given resident, and (b) allaying concerns of catastrophic data loss from their PDAs (e.g. by educating residents about procedures to recover information from PDA backup files).

(C) 2003 Elsevier Ireland Ltd. All rights reserved.

\section{Background}

We regularly observe medical house staff carrying hand-held devices, or personal digital assistants (PDAs), while on rounds, consulting with other staff, or writing patient summaries and this use is similar to previous reports [1-12]. At the time of this study, our institution's computerized hospital information systems included physician workstations, used primarily for order-entry and to access laboratory results, operative notes and other documentation. These workstations are situated throughout the hospital, usually at the perimeter of a ward or at a centralized workroom. To date, a few publications $[13,14]$ have evaluated perceived needs of residents; these focus on the uses of PDAs in a clinical setting, either in a primary care setting, such as family medicine or a specialty setting, such as an intensive care unit. As a first step towards understanding how PDAs might benefit our institution, we asked a convenience sample of our residents how they use or think they might like to use, PDAs. We asked these residents to fill in a brief survey of PDA usage, and then interviewed a sub sample of these residents to obtain more details on their PDA usage. Residents were selected from practices representing both primary care and specialty practices. These results, a systematic review of residents' perceived PDA needs, are important not only for exploring what these residents currently think about PDAs, but also for providing a foundation for further analysis of perceived needs in other health care groups (nurses, administrators) and actual usage by residents.

\section{Methods}

Our study used quantitative and qualitative research methods, incorporating both a survey and follow-up interviews with study participants. In addition to the quantitative survey data, we chose a descriptive or interpretative design [15] that allows categories and dimensions of analysis to emerge without making prior assumptions or hypotheses. These qualitative methods have long been used in education and sociology research on peoples' opinions and interests. Our study seeks to qualitatively understand what residents like and dislike about PDAs. Our initial survey allowed residents to tell us what they liked, disliked and how they used PDAs. The interviews were loosely structured to allow participants to describe issues of central importance to them.

Initially, we developed a survey to determine resident usage of PDAs. This web based survey made use of standard HTML forms objects, such as pull-down selection menus. This had the advantages of being quick to disseminate to the residents, and facilitated easy to analyze, structured, responses. We contacted all 223 residents (by e-mail) who were enrolled in the six residency programs; 88 (40\%) completed the survey between 26 October 2001 and 30 April 2002. The last set of questions in our survey allowed respondents to voluntarily provide contact information. Follow-up interviews were arranged with 15 residents who consented to be interviewed. These interviews were conducted between 24 April 2002 and 13 May 2002. The value of follow-up interviews became apparent when we evaluated the survey responses; because we designed the survey to be answered quickly, some responses were not detailed enough. In other cases, a follow-up interview provided an opportunity to fill in missing information, such as how residents react to the user interface of some commonly used medical applications (e.g. Patient Keeper and MedCalc). Below, we first describe the design of our survey, and then we describe our interview design. 
Table 1 Survey question topics

1. What medical practice are you in?

2. How often do you use a PDA?

3. If your answer to 2 was rare/never, why?

4. What kind of PDA?

5. What applications do you use now?

6. What application would you like to use?

7. May we contact you for follow-up questions?

\subsection{Survey development-question construction}

We designed our survey so that medical residents could answer it in less than $5 \mathrm{~min}$. We chose a web-based survey for the residents' convenience of accessing the survey as all our residents have Internet and e-mail access in the hospital and ambulatory clinic environments. Table 1 shows the question topics in this survey. The web pages consisted of a series of HTML forms; responses submitted through this form were subsequently downloaded and translated into a Microsoft ${ }^{\top M}$ Excel table for analysis. The final survey (along with the exact pull-down menus of answer options) may be viewed at the URL http: / / intercom.virginia.edu/SurveySuite / Surveys/pda.

\subsection{Survey development-resident selection}

Our survey participant selection criteria included practices: (1) both in specialist and primary care fields, and (2) some residency practices that strongly encouraged PDA usage and some practices that did not encourage PDA usage. Patton $[4,16]$ refers to this sampling method as purposeful sampling. We selected practices to represent both primary care (family medicine and internal medicine) as well as specialties (neurology, pediatrics, trauma surgery, and radiology). We selected family medicine practice because they strongly encouraged PDA usage: this practice provides PDAs to all incoming residents. Conversely radiology was selected because although the department tends to be technologically oriented, PDA usage is not specifically encouraged.

\subsection{Survey implementation}

We used telephone and e-mail to contact the residency programs directors of each of our identified practices (above). Directors were asked to send out an e-mail to all residents in their program requesting that they fill out our survey (we supplied them with an suggested text including the survey URL). Although, survey respondents were anonymous to us, because the survey asked respondents to indicate their residency program, response rates by program could be determined. Approximately 1-2 days after the initial e-mail to the residents, program directors were asked to send out a follow-up e-mail to encourage residents to fill out the survey.

\section{Design of structured interviews}

\subsection{Interview-question development}

Specific areas of interview questions were formulated after reviewing the responses from the surveyed residents. A series of questions were constructed for use in follow-up interviews. That is, the survey results informed us of topics where more detailed information was deemed useful. For example, the survey questionnaire responses did not tell us specifically what residents liked or disliked about using their PDAs (i.e. which parts of software were difficult to use or how often their PDAs actually failed). These interviews provided an opportunity to fill in this missing information. These interviews were structured in design: the same set of questions was asked of each resident in the same sequence. Table 2 contains the question topics for these interviews. A printed copy of these questions, in outline form was used at each interview, to guide the discussion.

\subsection{Interview-selection of interviewees}

All 24 residents who answered affirmatively that we could contact them for follow-up questions (and gave us contact information) were selected for possible interviews. Interview arrangements were made with 15 residents (the remaining nine residents did not respond to follow-up phone calls or were out of the locality at the time of contact). These residents represented a broad range of PDA experiences: two residents (one in radiology, one in internal medicine) were known to be early adopters of PDAs, two other residents had never used a PDA. Interviews were scheduled at times and locations convenient for the resident, and were conducted by one questioner (JB or JS) with one resident at a time.

\subsection{Interview-implementation}

The interview design included five sections: (1) consent and introduction, (2) collection of demo- 
Table 2 Question topics used in resident interviews

1. What year of residency are you currently?

2. Do you currently use a PDA?

3. Does your residency program encourage the use of PDAs?

4. What do you think are the top three advantages/disadvantages of PDAs?

If you currently use a PDA then ...

What kind of a PDA is it, who bought it, when?

Your most frequent three uses (for all three, list what you like and dislike about these uses)

If one of the top three uses is for medical calculators, then: under what circumstances do you use the medical calculator

If patient data is one of the uses, then: What kinds of patient data do you track/would you like to track?

I have a series of paper drawings of a hypothetical medical calculator, let's go through these drawings and let you comments on what you like and dislike. Also, please talk me through how you would try to use the features in each drawing.

I have a series of paper drawings of a hypothetical patient tracker, let's go through these drawings and let you comments on what you like and dislike. Also please talk me through how you would try to use the features in each drawing.

5. I have a series of paper drawings of a hypothetical medical calculator, let's go through these drawings and let you comments on what you like and dislike. Also please talk me through how you would try to use the features in each drawing.

6. I have a series of paper drawings of a hypothetical patient tracker, let's go through these drawings and let you comments on what you like and dislike. Also please talk me through how you would try to use the features in each drawing.

7. In an ideal world, how would you like to use your PDA?

graphic data, (3) discussion of likes and dislikes of PDAs and medical applications, (4) discussion of two medical applications, and (5) a summary including a chance to consider what applications would be preferred in an optimal environment. Table 2 presents the interview question topics.

Two of the questions involved discussing medical applications on PDAs, a medical calculator and a patient tracking application These applications appeared to us to be popular, as evidenced by casual observation of our residents' PDAs. These questions were designed to encourage residents to react to user interface issues and consider the practicality of these applications in their particular medical practices. Screenshots of the medical calculator application, MedCalc for Color Palm OS ${ }^{\circledR}$, were downloaded from the vendor's Internet web page, http://medcalc.med-ia.net/screen_color.html.

These were presented to the resident as the interviewer described selecting patients, choosing a calculation, and displaying results. We produced paper screenshots of the patient tracking application, Patient Keeper (Public Beta version 3.0, from Patient Keeper Inc.), by loading Patient Keeper into the personal computer application Palm OS ${ }^{\circledR}$ Emulator (version 3.0a8) from Palm Inc. and then printing out the displays of the Palm OS ${ }^{\circledR}$ window. Eight different screen displays showed the steps of using Patient Keeper with a hypothetical patient, includ- ing displaying a list of patients, a list of fourteen reports (such as prescriptions, diagnoses, laboratory results and procedure lists). The interviewer asked the resident for positive and negative impressions of these screen displays and how this application would or would not fit into their practices.

An audio tape recording was made of each interview and then transcribed (using the dictation software Dragon Naturally version 6.0 from Scan Soft Inc.). We used N6 (version 6, from QSR Ltd.) to organize and codify concepts occurring in the interview transcripts.

\section{Results}

\subsection{Survey results}

The response rates, shown in Table 3, varied between residency programs, from 100\% (trauma surgery and neurology) to $18 \%$ (pediatrics). A total of 88 residents submitted answers to the survey: of the residents that submitted contact information, 36 were male, 8 female ranging from first year residents through two chiefs of residency. The overall completion rate of $40 \%$ is comparable to other e-mail invitation, web-based surveys where all potential respondents have e-mail and Internet access [17]. 
Table 3 Survey response rates by specialty

\begin{tabular}{lll}
\hline Practice & $\begin{array}{l}\text { Number of } \\
\text { residents }\end{array}$ & $\begin{array}{l}\text { Number responding } \\
\text { (\% responding) }\end{array}$ \\
\hline Family medicine & 24 & $10(42)$ \\
Internal medicine & 84 & $22(26)$ \\
Neurology & 15 & $15(100)$ \\
Pediatrics & 34 & $6(18)$ \\
Radiology & 26 & $13(50)$ \\
Trauma surgery & 21 & $21(100)$ \\
\hline
\end{tabular}

Responses to the initial survey question about PDAs, "how often do you use your PDA?" are shown in Fig. 1. Most of the respondents use their PDAs on a daily basis: of all responding residents, more than $75 \%$ stated that they use their PDA on a daily basis; less than $15 \%$ of the respondents never use a PDA. The category "Infrequent" combines the survey responses "weekly," "monthly," or "rarely." Further, as shown in Fig. 2, a majority of responding residents in each surveyed program (except radiology) use PDAs on a daily basis. The category

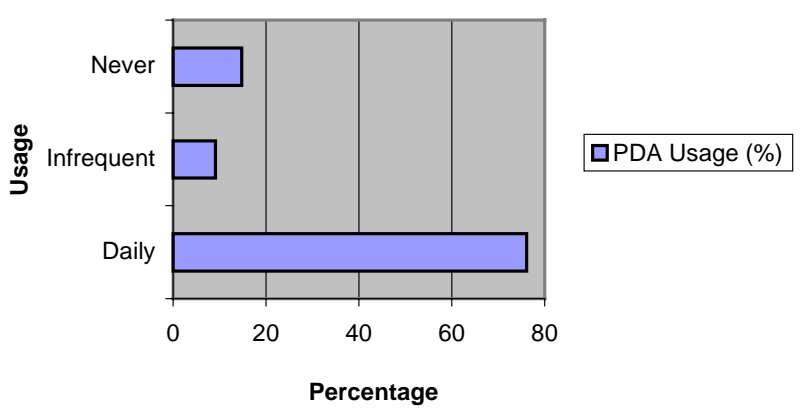

Fig. 1 PDA usage $(N=88)$.

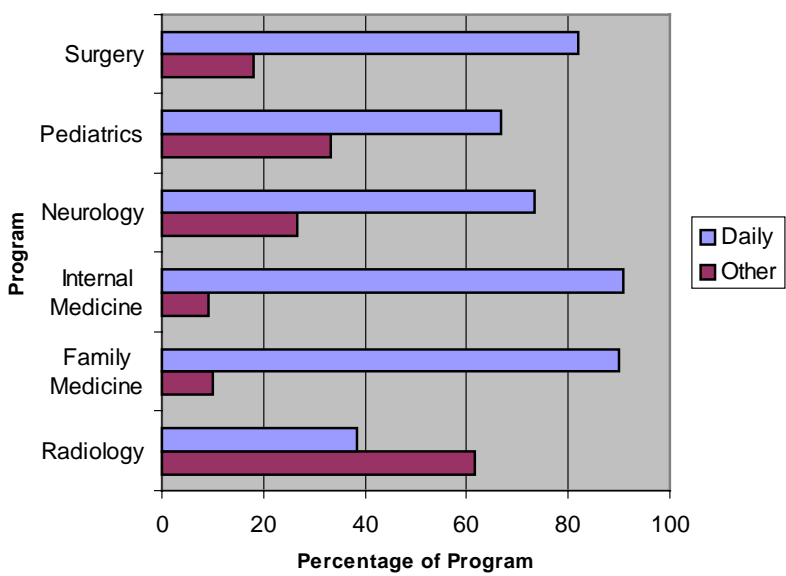

Fig. 2 PDA usage by residency program $(N=88$ residents).
Table 4 Applications currently used by residents

\begin{tabular}{ll}
\hline Application & $\begin{array}{l}\% \text { of all } \\
\text { respondents } \\
(n=88)\end{array}$ \\
\hline $\begin{array}{l}\text { Medical references (PDR: 5\%, } \\
\text { ePocrates }{ }^{\odot} \text { : 58\%, others: 21\%) }\end{array}$ & 84 \\
$\begin{array}{l}\text { Organizers, such as calendar, } \\
\text { phone book }\end{array}$ & 83 \\
$\begin{array}{l}\text { Medical calculators } \\
\text { Patient notes }\end{array}$ & 59 \\
Medical guidelines & 22 \\
$\quad$ InfoRetriever ${ }^{\odot}$ 12.5\%, Johns & 18 \\
$\quad$ Hopkins AntiMicrobial: 6\%, & \\
$\quad$ UVa AntiMicrobial: $0 \%)$ & \\
Patient management (Patient & 13 \\
$\quad$ Keeper: 6\%, other: $7 \%)$ & 0 \\
Medline & 0 \\
WebMD & 26 \\
Other &
\end{tabular}

"Other" includes all other available responses: "weekly," "monthly," "rarely," and "never."

The most popular current use of PDAs are medical reference applications, particularly the commercial product ePocrates ${ }^{\odot}$. The applications that were originally designed for PDAs; keeping a calendar, to do lists and other time management/organization tools, are close in popularity to the medical reference applications. Medical calculators are less popular: $59 \%$ of the residents stated they had used these types of applications. Some residents stated that they did keep some patient information on their PDA, using either informal notes (22\%) or commercially developed applications, such as Patient Keeper (13\%). Table 4 summarizes all of the currently used applications (Fig. 3).

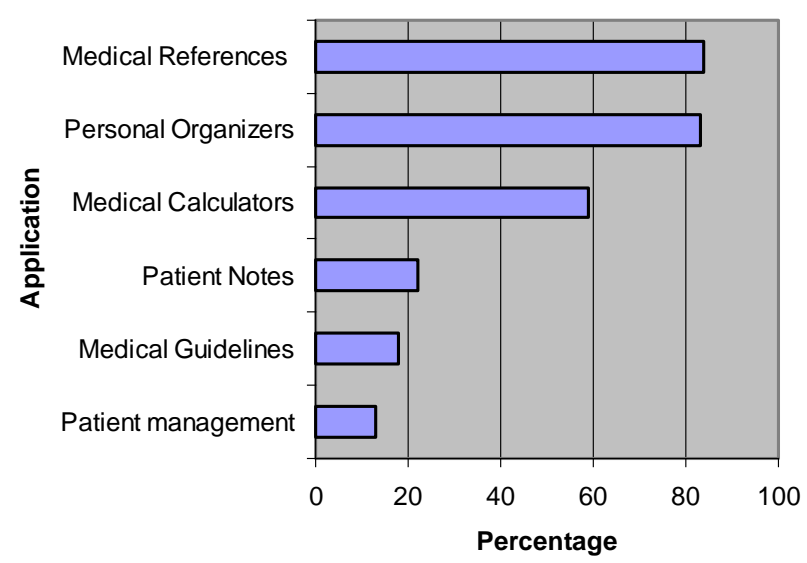

Fig. 3 Most popular currently used applications $(N=88$ residents). 


\begin{tabular}{ll}
$\begin{array}{l}\text { Table } 5 \text { Applications } \\
\text { learning }\end{array}$ & residents \\
\hline Application & $\begin{array}{l}\% \text { of all } \\
\text { respondents } \\
(n=88)\end{array}$ \\
\hline $\begin{array}{l}\text { Medical guidelines } \\
\text { (InfoRetriever }{ }^{\odot}: 28 \%, \text { Johns }\end{array}$ & 79.5 \\
Hopkins AntiMicrobial: $16 \%$, UVa & \\
AntiMicrobial: $35 \%)$ & \\
Patient management (WardWatch: & 45.5 \\
22\%, Patient Keeper: $21 \%$, & \\
others: $3 \%)$ & 42.0 \\
Medline & 31.8 \\
Medical reference (PDR: $12.5 \%$, & \\
ePocrates ${ }^{\odot}: 16 \%$, others: $\left.3 \%\right)$ & 30.7 \\
WebMD & 17.0 \\
Keep notes on my patients & 13.6 \\
E-mail & 8.0 \\
Organizers, such as calendar, & \\
phone book & 6.8 \\
Medical calculators & 8.0 \\
Other applications & \\
\hline
\end{tabular}

When asked about applications that they would be interested in learning to use, most residents wanted to learn about medical guidelines, particularly our institutions antimicrobial guidelines (see Tables 5 and 6). Between 30 and 40 percent of the respondents indicated an interest in using patient management, on-line journal access, or general medical reference applications.

\subsection{Interview results}

After an introduction, obtaining informed consent and demographic data, the initial interview topics

Table 6 Interview topics and common responses

\begin{tabular}{ll}
\hline Question/topic & Responses \\
\hline What are the top & 1. Speed \\
three advantages & 2. Organization \\
to a PDA? & $\begin{array}{l}\text { 3. Contact information } \\
\text { 4. Drug guidelines }\end{array}$ \\
What are the top & 1. Loss of data \\
three disadvantages & 2. Slow data entry \\
to a PDA? & 3. Physical size \\
& 4. Not enough drug \\
& information \\
What are your top & 1. Personal organization \\
three applications & 2. (Patient and hospital) \\
of a PDA? & contact information \\
& 3. Drug guidelines \\
& 4. Medical references \\
& 5. Medical calculators \\
\hline
\end{tabular}

focused on the overall likes and dislikes of PDAs by the residents. When asked "what are the top advantages of a PDA?", resident responses included: speed of getting information, ability to help organize the resident's professional calendar, contact information for either patients or colleagues, and drug information. Some residents felt they were less likely to lose information since it was all on their PDA and not on a collection of paper-based index cards, guideline pamphlets, and calendars. In cases where a resident indicated that they kept patient information on their PDA, the interviewer asked additional questions about the kind of patient information stored. In most cases, the patient information consisted of contact information (e.g. home phone numbers, cellular phone number, etc.). Two residents downloaded a list of hospital pager numbers and other internal phone numbers (e.g. the number of the phones next to various radiology reading rooms). One resident kept all computer access codes on their password-protected PDA.

We then asked, "What are your three most common applications?"' Responses included: personal organizers including calendars and address books, drug guidelines and medical references including ePocrates ${ }^{\odot}$, and InfoRetriever ${ }^{\odot}$, and medical calculators.

The final question on general PDA likes and dislikes was "What are the main disadvantages of using your PDA?' Responses included: data loss due to hardware or software failures, manual data entry is too slow, physical size (two residents indicated their Microsoft ${ }^{\top M}$ CE was too bulky, one respondent with a Palm V indicated the screen was too small), and respondents indicated they wanted more drug information (e.g. pediatric dosages, mechanisms of action) than was provided by the commercial pharmaceutical guidelines. Two of the respondents who talked about data loss mentioned a concern of becoming completely dependent on their PDA. One respondent worried that the ease of downloading journal articles might lead to a loss of library search skills. Two residents, while talking about data loss mentioned the cost of replacing a broken PDA. One resident, while discussing hardware failures, mentioned the cost of software as a barrier.

The next section of the interview consisted of the residents' impressions of demonstrations of a medical calculator and a patient tracking application. When presented with the walk-through of the medical calculator user interface, most residents reacted with indifference, that is, the residents did not seem to be interested or see any clear application to their work. Only two (of the seven residents with specific comments about this application) suggested they use a similar calculator and like having 
access to this PDA application. Most disliked the long list of formulas and would be more enthusiastic if they knew how to tailor the list to add their own calculations into the application. The residents with pediatrics experience wanted to have pediatric dosing calculations added to adult formulas.

Residents responded with indifference to the patient tracking user interface. Several residents commented that this application might be useful on a general service, inpatient environment, but that it did not seem useful for various specialties or in outpatient clinics.

The final interview question asked was, "In an ideal world, how would you like to use your PDA?"' Every resident answered this question with a detailed list of medical reports, particularly downloading data from our hospitals' Clinical Archive System (CAS), such as current lab results and discharge summaries. Four residents wanted to download some MIS (what does MIS refer to here?) and current medications. Two residents mentioned wishing to have specialist or nursing notes.

Of the two interviewed residents who did not have PDAs, one had recently broken their PDA (and hoped to eventually replace it), the other did not see a need in the current assignment of the Emergency Department (ED). In the follow-up discussion with the latter resident, the conversation focused on why PDAs were not useful in the ED setting, including: there was no need to look up drug information when an inexpensive pharmaceutical booklet would suffice, and patient tracking is currently handled by a system of paper logs and adhesive, colored labels. As this resident then thought through the usual routine, where the resident may leave the patient area to find a computer linked to CAS, the resident then concluded a PDA with CAS information on it would be beneficial during the patient interview.

Three residents mentioned the value of using a PDA in patient education. All three indicated that the patients appeared to like such usage: two of the residents said they believed the patients felt comforted by the use of such high technology. Another educational use of PDAs was mentioned by a radiology resident, who was able to locate a previously discussed relevant case during grand rounds.

\section{Discussion}

Despite significant improvements in hand-held computer hardware and software since Labkoff's 1995 Constellation Project study [1], our findings are comparable to this earlier research. Similar to this previous study, medical references and drug refer- ences were commonly used by residents, as were built-in application, such as address books, calendars, and to-do lists. Additionally, both studies mention physical size as a limitation to PDA usage, despite significant decreases in size and weight of current hand-held computer models. However, as memory has increased exponentially in the past 8 years (from $2 \mathrm{MB}$ on early hand-held computers to a maximum potential of $5 \mathrm{~GB}$ on current versions), the completeness of references was not mentioned as a major limitation among our participants. Concerns over security and data loss are new findings in our current study.

We selected departments to take our survey in a purposeful manner [16] representing the spectrum of practices both in terms of specialty versus primary care medicine, and in terms of whether the residents' teaching faculty specifically encouraged PDAs. For example, our family practice department represented not only a primary care setting, but also a practice where PDAs are strongly encouraged (each resident is provided one with the medical reference software, InfoRetriever ${ }^{\odot}$ (http: / / www. infopoems.com) pre-installed). Trauma surgery is another practice that strongly encourages PDA usage: the chief of the unit encourages residents each day to beam their summaries to the chiefs. On the other hand, the department of Radiology represents a technologically focused specialty where PDAs are not specifically encouraged or discouraged. In fact, two of the radiology residents that we interviewed commented that since they had easy access in radiology to a variety of clinical workstation computers, PDAs were best suited for general contact information: both information to contact their patients and information to contact other areas in the hospital (clinical laboratory phone numbers, pager numbers, etc.). Patient data, such as laboratory results and medication lists are already easily available and not needed on their PDAs. Both pediatrics and internal medicine are specialties where PDAs are neither in widespread use by the faculty nor are residents specifically encouraged or discouraged from using PDAs. Thus, we feel that departments contacted represent the range of medical practice types (specialist to primary care) and in acceptance of PDAs at our hospital.

We surveyed and interviewed both residents who currently used PDAs and residents who did not currently use PDAs. We asked the head of the residency programs to contact (by e-mail) all residents to inviting them to participate. One of the limitations of this approach is that there is more likely to be a selection bias towards more computer literate physicians. Computer novices would be less likely 
to either use a PDA, or fill out web-based surveys. This was considered to be less of a concern in our institution as all residents have web and e-mail access and are expected to use it daily for important communications. Two of the residents (one in radiology, the other in internal medicine) were chosen for interviews because they were known to be advocates of PDA usage. Two of the respondents had never used a PDA; we intentionally contacted both of these non-PDA users for interviews. Thus, while 75 of the 88 surveyed respondents (and 13 of 15 interviewed residents) are current PDA users, our results included the range of views from PDA advocates through PDA skeptics.

PDAs appear to be popular tools in our residency program: PDAs were used by most of the residents in every department we surveyed. Most use PDAs as part of their daily routine, both to check on evidence (through published references and practice guidelines) and to keep their daily schedules organized. Thus, the current usage of PDAs in our institution reflects the general design of PDAs - to provide a single information source for personal organization and general reference materials.

When we asked residents to speculate on how PDAs might optimally help them, the answers tended to focus on getting information for that resident's current needs, such as patient laboratory results and discharge summaries. That is, when reflecting about future PDA usage, many residents talked about being able to use PDAs to obtain and transmit information about patients under their care currently; to obtain information, such as laboratory results, medications, and discharge summaries. This strong interest in having PDAs with information about their own patients, contrasts with the mild interest in the medical calculator and patient tracking applications shown to interviewees. We speculate that if our patient tracker walk-through contained some indication that the application could be tailored to the residents' current patients, interest would have been much higher.

The fact that PDAs are used in all surveyed departments, including those departments where, based on our casual observation, there is neither great PDA usage by the faculty nor do the faculty specifically encourage PDA usage suggests that residents are independently choosing to use PDAs. In fact the names of two residents were cited as encouraging PDA usage, either by providing useful software or simply being role models. Residents appear to be teaching each other about PDAs, and residents are demonstrating their usage in grand rounds and other teaching sessions. We speculate that PDA usage may very well continue to rise.
One of the main drawbacks of PDAs is that of developing an absolute dependency on devices that can catastrophically fail or can easily be lost. This drawback most likely needs to be confronted before PDAs can have widespread acceptance. This is an issue of perception as much as reality: for example, out of the 18 interviewed residents, just two residents had their PDA crash and apparently lose data; the PDA for a third resident was physically broken just before starting to use it. Even though no more than three of the 18 interviewed residents had actually experienced catastrophic data loss, every interviewed resident currently using a PDA mentioned a concern of breaking the PDA or losing data. We suggest that regardless of the frequency of PDA disasters actually occurring, residents will be much more willing to use PDAs if they can be shown basic protection, such as how to recover data, prevent catastrophic power loss, and perform regular file synchronization between PDA and desktop computer.

Issues related to data security and confidentiality were mentioned: some residents use their PDA to store patient information, which pose compliance issues related to privacy and security rules passed in the Health Information Portability and Accountability Act of 1996, generally known as HIPAA, which require health care workers to keep patient identification information private. At least four interviewed residents store patient contact information (phone numbers, addresses, e-mail addresses, etc.), usually in conjunction with information about contacting various services within the hospital (radiology reading room phone number, laboratory phones). Further, many residents when speculating about how they might like to use a PDA, wanted to include CAS or medical record information on their PDA. The residents keeping patient contact information do attempt to secure this information either by password-protecting any use of the PDA, by obscuring the data in files, or making the files accessible only to one PDA user. While more rigorous security will be required, at the same time the ability for a resident to use a PDA for contact information is very useful. Two of the residents using PDAs for patient contact information enthusiastically endorsed this application. These residents commented that the PDA made their ability to follow-up on patients much more likely and they were able to hold a much more effective conversation with the patient.

\section{Conclusions}

Results of our survey and interviews identified several specific advantages and disadvantages of PDA 
usage by residents that merit further study. Advantages included: (1) many residents readily adapted the personal organizers (calendars, address books, to-do lists) to keep track of their meetings and daily professional tasks, stay in touch with patients, and (2) medical references (such as ePocrates ${ }^{\odot}$ ) are used most by the surveyed residents to answer immediate medical questions. Also, residents were very interested in the possibilities of including their own current clinical data particularly laboratory results and daily discharge summaries. Perceived drawbacks include: (1) current commercial medical applications, such as calculators and patient trackers that do not appear to the residents as useful in their methods of practice, e.g. to tailor lists of calculations to just those actually used, (2) physical size (both too small a display size, and too bulky overall), and (3) several residents mentioned a concern of becoming too dependent on one source of information, a source that was viewed as being too easily to lost or broken.

Two broad themes emerged. First, most residents use PDAs daily, regardless of practice or whether their program encourages PDAs. That is, since residents in all seven of our surveyed practices use PDAs and most of them use PDAs on a daily basis; we conclude that PDAs are being widely used across the spectrum of primary care and specialty practices, and regardless of whether a residency program specifically encourages PDA usage. Second, security and HIPAA compliance issues need to be addressed, in part by resident education about securing PDA files properly. This important issue merits further investigation to see if this is a widespread problem. As an extension of these two themes, PDAs may become even more widely used if two aspects can be addressed: (a) providing secure clinical data specific to an individual resident on their PDAs, and (b) allaying concerns of catastrophic data loss from their PDAs (e.g. by educating residents about procedures to recover information from PDA backup files). Overall, these three themes suggest that while PDAs are in fairly widespread use among residents, issues of security and data loss now need to be addressed if PDAs are to be fully utilized.

Future directions of this work include identifying information seeking behavior of residents and studying how PDAs are currently used for information seeking, and then considering ways PDAs might enhance information seeking and gathering in the future. We intend to directly observe residents as they perform various duties including: both inpatient and outpatient care, as well as during research or educational periods (such as grand rounds and preceptor-resident sessions). We will also ex- plore with the residents how they might use PDAs if their device contained clinical labs, summary reports, and other patient-specific data. Further, we expect that the results of these surveys and interviews will help future explorations of potential PDA needs with other health care professionals, such as attending physicians, nurses, administrators, and other clinical staff.

\section{Acknowledgements}

The interview and survey methods in this research were approved by the University of Virginia Institutional Review Board for Social and Behavioral Sciences, Project\# 2001-0310-00.

\section{References}

[1] S.E. Labkoff, S. Shah, J. Bormel, Y. Lee, R.A. Greenes, The Constellation Project: experience and evaluation of personal digital assistants in the clinical environment, in: Proceedings of the 19th Annual Symposium on Computer Applications in Medical Care, 1995, pp. 678-682.

[2] Anonymous, Physician's use of hand-helds increases from $15 \%$ in 1999 to $26 \%$ in 2001: Harris interactive poll results, Harris Poll. 8-24-2002 (electronic citation).

[3] American College of Physicians, ACP-ASIM survey finds nearly half of U.S. members use hand-held computers: ACP-ASIM press release, American College of Physicians, 9-3-2002 (electronic citation).

[4] D.F. Criswell, M.L. Parchman, Hand-held computer use in us family practice residency programs, J. Am. Med. Inform. Assoc. 9 (1) (2002) 80-86.

[5] T.S. Alderson, N.T. Oswald, Clinical experience of medical students in primary care: use of an electronic log in monitoring experience and in guiding education in the Cambridge community based clinical course, Med. Educ. 33 (6) (1999) 429-433.

[6] S.B. Bird, R.S. Zarum, Is ED resident patient care documentation increased with the use of hand-held computerized devices? Acad. Emerg. Med. 8 (5) (2001) 492 (abstract).

[7] R. Garvin, F. Otto, D. McRae, Using hand-held computers to document family practice resident procedure experience, Fam. Med. 32 (2) (2000) 115-118.

[8] A.L. Helwig, C. Flynn, Using palm-top computers to improve students' evidence-based decision making, Acad. Med. 73 (5) (1998) 603-604.

[9] A.F. Jerant, Training residents in medical informatics, Fam. Med. 31 (7) (1999) 465-472.

[10] R.L. Krippendorf, D.E. Simpson, D. Schiedermayer, Promoting reflective teaching with personal digital assistants, Acad. Med. 74 (5) (1999) 577.

[11] T.K. Malan, W.H. Haffner, A.Y. Armstrong, A.J. Satin, Hand-held computer operating system program for collection of resident experience data, Obst. Gynecol. 96 (5 Pt. 1) (2000) 792-794.

[12] C.F. Pipas, P.A. Carney, M.S. Eliassen, S.C. Mengshol, L.H. Fall, A.L. Olson, K.E. Schifferdecker, M.T. Russell, D.A. Peltier, D.W. Nierenberg, Development of a hand-held computer documentation system to enhance an integrated primary care clerkship, Acad. Med. 77 (7) (2002) 600-609. 
[13] B.W. Beasley, Utility of palmtop computers in a residency program: a pilot study, South. Med. J. 95 (2) (2002) 207211.

[14] G. Rao, Introduction of hand-held computing to a family practice residency program, J Am. Board. Fam. Pract. 15 (2) (2002) 118-122.
[15] Tesch, Renata, Qualitative Research: Analysis Types and Software Tools, Falmer Press, 1990.

[16] M.Q. Patton, Qualitative Evaluation Methods, Sage, Beverly Hills, 1980.

[17] P. Couper Mick, Web surveys: a review of issues and approaches, Public Opin. Q. 64 (2003) 464-494.

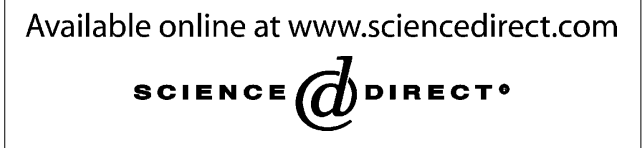

